\title{
CENTRAL LIMIT THEOREM ON CHEBYSHEV POLYNOMIALS
}

\author{
Young-Ho AhN
}

\begin{abstract}
Let $T_{l}$ be a transformation on the interval $[-1,1]$ defined by Chebyshev polynomial of degree $l(l \geq 2)$, i.e., $T_{l}(\cos \theta)=\cos (l \theta)$. In this paper, we consider $T_{l}$ as a measure preserving transformation on $[-1,1]$ with an invariant measure $\frac{1}{\pi \sqrt{1-x^{2}}} d x$. We show that If $f(x)$ is a nonconstant step function with finite $k$ discontinuity points with $k<l-1$, then it satisfies the Central Limit Theorem. We also give an explicit method how to check whether it satisfies the Central Limit Theorem or not in the cases of general step functions with finite discontinuity points.
\end{abstract}

\section{INTRODUCTION}

Let $(X, \mu)$ be a probability measure space. A measurable transformation $T: X \rightarrow$ $X$ is said to be measure preserving if $\mu\left(T^{-1} E\right)=\mu(E)$ for every measurable subset $E$. A measure preserving transformation $T$ on $X$ is called ergodic if $f(T x)=f(x)$ holds only for constant functions and it is called weakly mixing if the constant function is the only eigenfunction with respect to $T[3,5]$.

Let $\mathbf{1}_{E}$ be the characteristic function of a set $E$ and consider the behavior of the sequence $\sum_{k=0}^{n-1} \mathbf{1}_{E}\left(T^{k} x\right)$ which equals the number of times that the points $T^{k} x$ visit $E$. The Birkhoff Ergodic Theorem applied to the ergodic transformation $\left(\frac{1}{2}, \frac{1}{2}\right)$ Bernoulli shift on $\prod_{k=0}^{\infty}\{0,1\}$ gives the Laws of the Large Numbers.

Let $T$ be a transformation which is piecewise expanding on the unit interval $X=[0,1)$ and $g(x) \equiv \frac{1}{\left|T^{\prime}(x)\right|}$ be a function of bounded variation, where $T^{\prime}(x)$ is the appropriate one-sided derivative at the discontinuities. Then it is well-known that there exists an absolutely continuous invariant measure with respect to the Lebesgue measure. Furthermore if $T$ is weakly mixing with respect to the $T$-invariant absolutely continuous measure, $f(x)$ is a bounded variation function and the functional

Received by the editors August 14, 2014. Revised October 10, 2014. Accepted Oct. 10, 2014. 2010 Mathematics Subject Classification. 28D05, 37A50, 47A35.

Key words and phrases. Chebyshev polynomials, the central limit theorem, measure preserving, ergodic, weakly mixing, bounded variation function. 
equation

$$
f=g \circ T-g+c
$$

does not have any solution $g(x)$ for any constant $c \in \mathbb{R}$, then we can apply the Central Limit Theorem to the function $f(x)[2]$.

For each natural number $l(l \geq 2)$, let $T_{l}$ be the transformation on the interval $[-1,1]$ defined by Chebyshev polynomial of degree $l$. In this paper, we consider $T_{l}$ as a measure preserving transformation on $[-1,1]$ with an invariant measure $\frac{1}{\pi \sqrt{1-x^{2}}} d x$. We show that if $f(x)$ is a step function with finite $k$-discontinuity $\operatorname{points}(k<l-1)$ then it satisfies the Central Limit Theorem. We also give a explicit method how to check whether it satisfies the Central Limit Theorem or not in the cases of general step functions with finite discontinuity points. It is known that the entropy of $\left([-1,1], \frac{1}{\pi \sqrt{1-x^{2}}} d x, T_{l}\right)$ is $\log l$ for each $l \geq 2[1]$.

\section{Properties of Chebyshev Polynomials}

Let $T_{l}$ be the Chebyshev polynomial of degree $l(l \geq 2)$. Recall that $T_{l}$ is defined by

$$
T_{l}(\cos x)=\cos (l x)
$$

on $[-1,1]$. Chebyshev polynomials are orthogonal in the Hilbert space

$$
H=L^{2}([-1,1], \rho(x) d x)
$$

where

$$
\rho(x)=\frac{1}{\pi \sqrt{1-x^{2}}} .
$$

Let $T:(X, \mu) \rightarrow(X, \mu)$ and $\Lambda:(Y, \nu) \rightarrow(Y, \nu)$ be measure preserving. Two measure preserving transformations are said to be measure theoretically isomorphic if there exists an isomorphism $\psi:(X, \mu) \rightarrow(Y, \nu)$ such that $\psi \circ T=\Lambda \circ \psi$, in other words, the following diagram commutes:

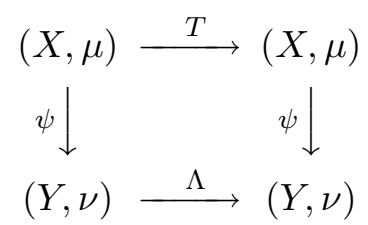

From now on, let $\nu$ be the Lebesgue measure on $[0,1]$ and $\mu$ be an absolutely continuous measure on $[-1,1]$ with the density function $\rho(x)$. i.e., the measure $\mu$ is defined by

$$
\mu(E)=\int_{E} \rho(x) d x
$$


Definition 1. For each $l \in \mathbb{N}$, let $\Lambda_{l}$ be a map on $[0,1]$ defined by

$$
\Lambda_{l}(x)= \begin{cases}l x-k, & \text { if } k \text { is even, } \\ k+1-l x, & \text { if } k \text { is odd }\end{cases}
$$

for $\frac{k}{l} \leq x \leq \frac{k+1}{l}, k=0,1, \cdots, l-1$.

It is well-known that $\Lambda_{l}$ preserves the Lebesgue measure $\nu$ and it is weakly mixing.

Lemma 1. Let $T_{l}$ be the $l$-th Chebyshev polynomial of order $l \geq 2$. Then $T_{l}$ preserves the measure $\mu$ on $([-1,1])$ and is measure theoretically isomorphic to the transformation $\Lambda_{l}$ on $([0,1], \nu)$ by a topological homeomorphism $\psi(x)=\frac{1}{\pi} \arccos (x)$.

Proof. Let $\phi(y)$ be the inverse function of $\psi(x)$, i.e., $\phi(y)=\cos (\pi y)$ from $[0,1]$ to $[-1,1]$. It is obvious that $\phi \circ \Lambda_{l}=T_{l} \circ \phi$ holds. Hence $\psi \circ T_{l}=\Lambda_{l} \circ \psi$. So it is enough to show that $\phi$ is a measure theoretical isomorphism. Note that the inverse image of $[\phi(y), 1]$ under $\phi$ is $[0, y]$, which has Lebesgue measure equal to $y$. For $\phi$ to be an measure theoretical isomorphism, it must satisfy

$$
\mu([\phi(y), 1])=y
$$

for all $0 \leq y \leq 1$. Thus $\mu([x, 1])=\psi(x)$ and $\mu([0, x])=1-\psi(x)$ for all $-1 \leq x \leq 1$, because $\mu$ is a probability measure on $[-1,1]$. Since

$$
\frac{d}{d x}\left(\mu([0, x])=\frac{d}{d x}(1-\psi(x))=\frac{1}{\pi \sqrt{1-x^{2}}}=\rho(x),\right.
$$

$\phi$ is a isomorphism and the following diagram commutes.

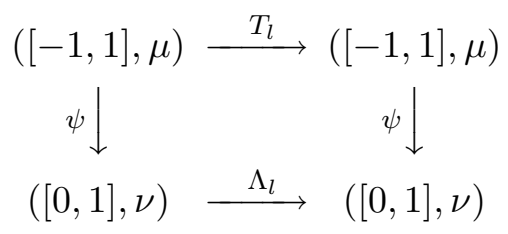

Hence $T_{l}$ is a measure preserving transformation on $([-1,1], \mu)$ and weakly mixing.

\section{The Central Limit Theorem}

The following lemma gives a sufficient condition for a special class of transformations on which the Central Limit Theorem holds [2]. In Lemma 2, $\mu$ is an arbitrary absolutely continuous measure. 
Lemma 2. Let $T$ be a piecewise continuously differentiable and expanding transformation on an interval $[a, b]$, i.e., there exists a partition

$$
a=a_{0}<a_{1}<\cdots<a_{k-1}<a_{k}=b
$$

such that $T$ is continuously differentiable on each $\left[a_{i-1}, a_{i}\right](1 \leq i \leq k)$ and $\left|T^{\prime}(x)\right|>$ $B$ for some constant $B>1$ (At the endpoints of an interval we consider directional derivatives). Assume that $\frac{1}{\left|T^{\prime}(x)\right|}$ is a function of bounded variation. Suppose that $T$ is weakly mixing with respect to an invariant probability measure $\mu$. Let $f(x)$ be a function of bounded variation such that the equation

$$
f(x)=g(T x)-g(x)+c,
$$

where $c$ is constant, has no solution $g(x)$ of bounded variation. Then

$$
\sigma^{2}=\lim _{n \rightarrow \infty} \int_{0}^{1}\left(\frac{S_{n} f-n \mu(f)}{\sqrt{n}}\right)^{2} d \mu>0
$$

and, for every $\alpha$,

$$
\lim _{n \rightarrow \infty} \mu\left\{x: \frac{S_{n} f(x)-n \mu(f)}{\sigma \sqrt{n}} \leq \alpha\right\}=\frac{1}{\sqrt{2 \pi}} \int_{-\infty}^{\alpha} \exp \left(-t^{2} / 2\right) d t,
$$

where

$$
S_{n} f(x)=\sum_{j=0}^{n-1} f\left(T^{j}(x)\right)
$$

and

$$
\mu(f)=\int_{0}^{1} f(x) d \mu(x) .
$$

Since $T_{l}$ is measure theoretically isomorphic to $\Lambda_{l}$ by a topological homeomorphism, we may assume that the transformation $T_{l}$ on $([-1,1], \mu)$ satisfies all the conditions of Lemma 2.

Proposition 1. For the measure preserving transformation $T_{l}$ on $[-1,1]$ defined by $l$-th Chebyshev polynoimal, if an $\mathbb{R}$-valued function $f(x)$ is a step function with finite discontinuity points and $f(x)=g\left(T_{l} x\right)-g(x)+c$ with a constant $c$, then $g(x)$ is also a step function with finite discontinuity points.

Proof. Recall that the measure preserving transformation $T_{l}$ on $([-1,1], \mu)$ and the measure preseving transformation $\Lambda_{l}$ on $([0,1], \nu)$ are measure theoretically isomorphic via the topological homeomorphism $\psi(x)=\frac{1}{\pi} \arccos (x)$ by Lemma 1. As in Lemma 1 , let $\phi(y)=\cos (\pi y)$ be the inverse function of $\psi(x)$. Note that $f(x)$ is a 
step function with finite discontinuity points if and only if $f(\phi(y))$ is a step function with finite discontinuity points. Furthermore the functional equation

$$
f(x)=g\left(T_{l} x\right)-g(x)+c
$$

has a solution if and only if the functional equation

$$
f(\phi(y))=g\left(T_{l}(\phi(y))\right)-g(\phi(y))+c
$$

has a solution. Let $v$ be the variation of $f(x), F(y)=\exp \left(-2 \pi i \frac{1}{v} f(\phi(y))\right) \times$ $\exp \left(2 \pi i \frac{1}{v} c\right)$ and $G(y)=\exp \left(2 \pi i \frac{1}{v} g(\phi(y))\right)$. Note that the number of discontinuity points of $f(x)$ is equal to the number of discontinuity points of $F(y)$ and if the functional equation

$$
f(\phi(y))=g\left(T_{l}(\phi(y))\right)-g(\phi(y))+c
$$

has a solution then the functional equation

$$
F(y) G\left(\Lambda_{l} y\right)=G(y)
$$

has a solution. So it is enough to show that $G(y)$ is also a step function with finite discontinuity points, because if $g(\phi(y))$ is a bounded variation function and $G(y)=\exp \left(2 \pi i \frac{1}{v} g(\phi(y))\right)$ is a step function with finite discontinuity points, then $g(\phi(y))$ also has to be a step function with finite discontinuity points. For the notational simplicity, we will prove the proposition in the case $l=2$.

Let $\mathcal{P}$ be a partition of [0, 1] defined by $\mathcal{P}=\left\{\left[0, \frac{1}{2}\right),\left[\frac{1}{2}, 1\right]\right\}$, and $\mathcal{P}_{N}=\bigvee_{k=0}^{N-1} T_{2}^{-k} \mathcal{P}$. Let $D=\{z \mid F(y)$ is discontinuous at $y=z\}, m$ be the cardinality of discontinuity $D$ and $D_{\epsilon}$ be the $\epsilon$-neighborhood of $D$, i.e., $D_{\epsilon}=\left\{\bigcup_{z \in D}(z-\epsilon, z+\epsilon)\right\}$. Then there exists $\epsilon_{0}$ such that for all $0<\epsilon<\epsilon_{0}, \nu\left(D_{\epsilon}\right)=2 m \epsilon$. Now choose an integer $N$ such that $\left(\frac{1}{2}\right)^{N}<\epsilon_{0}$ and $m \cdot\left(\frac{1}{2}\right)^{N-1}<\frac{1}{2}$.

If $I \in \mathcal{P}_{N}$ and if $I \cap D \neq \phi$, then $I \subset D_{\epsilon}$ for $\epsilon=\left(\frac{1}{2}\right)^{N}$. Hence the totality of $I \in \mathcal{P}_{N}$ with $I \cap D \neq \phi$ measures at most $2 m \cdot\left(\frac{1}{2}\right)^{N}$. By the similar argument, the totality of $I \in \mathcal{P}_{N+j}, j \geq 0$ such that $I \cap D \neq \phi$ measures at most $2 m \cdot\left(\frac{1}{2}\right)^{N+j}$.

Fix $L>0$ and consider the collection of $I \in \mathcal{P}_{N+L}$ having the property that $T^{j} I \cap D \neq \phi$ for some $0 \leq j \leq L-1$. Since $T^{j} I \in \mathcal{P}_{N+L-j}$ for these $j$, and $\Lambda_{2}$ is Lebesgue measure preserving, these intervals have the total Lebesgue measure at most

$$
\sum_{j=1}^{L-1} 2 m \cdot\left(\frac{1}{2}\right)^{N+L-j} \leq m \cdot\left(\frac{1}{2}\right)^{N-1} \leq \frac{1}{2}
$$


Let $Q(N, L)$ be the sub-collection of $\mathcal{P}_{N+L}$ such that $T^{j} I \cap D=\phi$ for all $0 \leq$ $j \leq L-1$. Then for each $I \in Q(N, L), F(y) F\left(\Lambda_{2} y\right) \cdots F\left(\Lambda_{2}^{L-1} y\right)$ is constant, say $\lambda_{I, L}$ with $\left|\lambda_{I, L}\right|=1$. Since $G(y)=F(y) G\left(\Lambda_{2} y\right), G(y)=F(y) F\left(\Lambda_{2} y\right) \cdots F\left(\Lambda_{2}^{L-1} y\right) G\left(\Lambda_{2}^{L} y\right)$. Hence $G(y)=\lambda_{I, L} \cdot G\left(\Lambda_{2}^{L} y\right)$ holds almost everywhere on $I$. Letting $\Lambda_{2}^{L} I=J \in \mathcal{P}_{N}$, the map $\Lambda_{2}^{L}: I \rightarrow J$ is bijective and it is easily shown that

$$
\left|\frac{1}{\nu(I)} \int_{I} G(y) d \nu(y)\right|=\left|\frac{1}{\nu(J)} \int_{J} G(z) d \nu(z)\right| \cdot
$$

Since $Q(N, L)$ measures at least $\frac{1}{2}$, the set of $y$ which is interior to some $I \in$ $Q(N, L)$ for an infinitely number of $L$ must also measures at least $\frac{1}{2}$. Fixing such an $y$, we have that (1) holds. We may assume that $y$ is also a Lebesgue point of $G$. Since $\mathcal{P}_{N}$ is finite, it can be assumed $J$ is always the same on the right side of (1). By the Lebesgue density theorem[4], we can assume that the left side of (1) tends to $G(y)$. Hence

$$
\left|\frac{1}{\nu(J)} \int_{J} G(z) d \nu(z)\right|=|G(y)|=1 .
$$

Since $|G(z)|=1$ for all $z \in[0,1], G(z)$ has to be constant on $J$. Since $F(y)$ is a step function with finite discontinuity and $\Lambda_{2}^{N} J=[0,1], G(y)$ is also a step function with finite discontinuity. Hence the conclusion follows.

Theorem 1. Let $T_{l}$ be a measure preserving transformation on $\left([-1,1], \frac{1}{\pi \sqrt{1-x^{2}}} d x\right)$ defined by Chebyshev polynomial of degree $l(l \geq 2)$. If $f(x)$ is a nonconstant step function with finite $k$-discontinuity points with $k<l-1$ then it satisfies the Central Limit Theorem, i.e.,

$$
\sigma^{2}=\lim _{n \rightarrow \infty} \int_{0}^{1}\left(\frac{S_{n} f-n \mu(f)}{\sqrt{n}}\right)^{2} d \mu>0
$$

and, for every $\alpha$,

$$
\lim _{n \rightarrow \infty} \mu\left\{x: \frac{S_{n} f(x)-n \mu(f)}{\sigma \sqrt{n}} \leq \alpha\right\}=\frac{1}{\sqrt{2 \pi}} \int_{-\infty}^{\alpha} \exp \left(-t^{2} / 2\right) d t,
$$

where $S_{n} f(x)=\sum_{j=0}^{n-1} f\left(T^{j}(x)\right), d \mu=\frac{1}{\pi \sqrt{1-x^{2}}} d x$ and $\mu(f)=\int_{0}^{1} f(x) d \mu(x)$.

Proof. It is enough to show that the functional equation

$$
f(x)=g\left(T_{l} x\right)-g(x)+c
$$

has no solution. Suppose it is not, by Proposition $1, g(x)$ is also a step function with finite discontinuity points. Hence $g(x)$ can be expressed as 


$$
g(x)=\sum_{j=0}^{m-1} c_{j} \mathbf{1}_{\left[a_{j}, a_{j+1}\right]}(x)
$$

where $-1=a_{0}<a_{1}<\cdots<a_{m}=1$. Since $g(x)$ has $m-1$ discontinuity points, $g\left(T_{l} x\right)$ has at least $l(m-1)$ discontinuity points and $g\left(T_{l} x\right)-g(x)+k$ has at least $(l-1)(m-1)$ discontinuity points. Since $f(x)$ has $k$ discontinuity points, we have

$$
0 \leq m-1 \leq \frac{k}{l-1} .
$$

So if $k<l-1$ then $m$ has to be 1 and $g(x)$ has to be a constant function. It is a contradiction to the assumption that $f(x)$ is not a constant function.

Theorem 2. Let $T_{l}$ be a measure preserving transformation on $\left([-1,1], \frac{1}{\pi \sqrt{1-x^{2}}} d x\right)$ defined by Chebyshev polynomial of degree $l(l \geq 2)$. If $f(x)$ is a nonconstant step function with finite discontinuity points and $f(x)$ is constant on the interval $\left[-1, \cos \left(\frac{l-1}{l} \pi\right)\right]$, then it satisfies the Central Limit Theorem.

Proof. Letting $J=\left[-1, \cos \left(\frac{l-1}{l} \pi\right)\right]$, we have $T_{l}(J)=[-1,1]$. Suppose there exists an function $g(x)$ which satisfies the functional equation,

$$
f(x)=g\left(T_{l} x\right)-g(x)+c .
$$

By Proposition 1, there exists $x_{1}$ such that $g(x)$ is constant on $\left[-1, T_{l}\left(x_{1}\right)\right] \supset\left[-1, x_{1}\right]$. If we take any $x \in\left[-1, x_{1}\right]$, then both $x$ and $T_{l}(x)$ are in $\left[-1, x_{1}\right]$ and $g\left(T_{l}(x)\right)=g(x)$. Since $f(x)=g\left(T_{l} x\right)-g(x)+c$, we have $f(x)=c$ for all $x \in J$. Therefore $g\left(T_{l}(x)\right)=$ $g(x)$ for all $x \in J$, and $g(x)=g(-1)$ for all $x \in\left[-1, T_{l}\left(x_{1}\right)\right]$. If $T_{l}\left(\left[-1, x_{1}\right]\right)=[-1,1]$, then $g(x)$ has to be a constant function and $f(x)$ also has to be constant. it completes the proof. Otherwise, letting $x_{2}=T_{l}\left(x_{1}\right)$, we have $g(x)$ is a constant on $T_{l}\left(\left[-1, x_{2}\right)\right)$ by exactly the same argument by using $x_{2}$ in the place of $x_{1}$. Iterating this argument if we need it, we get $g(x)$ is constant and the conclusion follows.

In the following Proposition, we give an explicit method how to check whether it satisfies the Central Limit Theorem or not in the cases of general step functions with finite discontinuity points. For the simplicity, we consider the case $l=2$ and $f(x)$ is a step function with 1 or 2 discontinuity points.

Proposition 2. Let $T_{2}$ be a measure preserving transformation on $\left([-1,1], \frac{1}{\pi \sqrt{1-x^{2}}} d x\right)$ defined by Chebyshev polynomial of degree 2. If $f(x)$ is a step function with finite $k$-discontinuity points with $k \leq 2$, then it satisfies the Central Limit Theorem with respect to $T_{2}$ except for the functions of the form 


$$
f(x)=b \cdot \mathbf{1}_{\left[-1,-\frac{1}{2}\right]}(x)-b \cdot \mathbf{1}_{\left[-\frac{1}{2}, \frac{1}{2}\right]}(x)+c
$$

with some constants $b, c$.

Proof. As in the proof of Proposition 1, let $\phi(y)=\cos (\pi y)$. Then the functional equation

$$
f(x)=g\left(T_{l} x\right)-g(x)+c
$$

has a solution if and only if the functional equation

$$
f(\phi(y))=g\left(T_{l}(\phi(y))-g(\phi(y))+c\right.
$$

has a solution. Furthermore $F(y)=f(\phi(y))$ has the same discontinuity points as $f(x)$.

Case 1) Suppose that $f(x)$ has 1-discontinuity point, i.e., $F(y)=f(\phi(y))$ has the form of

$$
F(y)=b_{0} \cdot \mathbf{1}_{[0, d]}(y)+b_{1} \cdot \mathbf{1}_{[d, 1]}(y)
$$

and $G(y)=g(\phi(y))$ is the solution of the functional equation $F(y)=G\left(\Lambda_{2} y\right)-$ $G(y)+c$, then $G(y)$ can be expressed as

$$
G(y)=\sum_{j=0}^{m-1} c_{j} \mathbf{1}_{\left[a_{j}, a_{j+1}\right]}(y)
$$

where $0=a_{0}<a_{1}<\cdots<a_{m}=1$. By exactly the same argument as in the proof of Theorem 1 , we have $m=1$ or 2 . When $m=1, G(y)$ has to be constant, and $f(x)$ also has to be constant. It is a contradiction. When $m=2$, then $G(y)$ has the form of $G(y)=c_{0} \mathbf{1}_{[0, a]}(y)+c_{1} \mathbf{1}_{[a, 1]}(y)$ with some constants $c_{0}, c_{1}$ and constant $0<a<1$. Since both $G(y)$ and $G\left(\Lambda_{2} y\right)$ have the same value on the interval $[0, a / 2]$, we have $b_{0}=c$. Integrating the functional equation

$$
F(y)=G\left(\Lambda_{2} y\right)-G(y)+c,
$$

we get a equation $d c+(1-d) b_{1}=c$. Hence $b_{1}=c$ and $f(x)$ is constant. It is a contradiction to the assumption of $f(x)$. Thus if $f(x)$ has 1-discontinuity point, then it satisfies the Central Limit Theorem.

Case 2) Suppose that $f(x)$ has 2-discontinuity points. Then $F(y)=f(\phi(y))$ has the form of

$$
F(y)=b_{1} \cdot \mathbf{1}_{\left[0, d_{1}\right]}(y)+b_{2} \cdot \mathbf{1}_{\left[d_{1}, d_{2}\right]}(y)+b_{3} \cdot \mathbf{1}_{\left[d_{2}, 1\right]}(y) .
$$

As in the case 1 , letting $G(y)$ be a solution of the functional equation $F(y)=$ $G\left(\Lambda_{2} y\right)-G(y)+c$, we have $G(y)=\sum_{j=0}^{m-1} c_{j} \mathbf{1}_{\left[a_{j}, a_{j+1}\right]}(y)$ with $m=2$ or 3 . When 
$m=2$, the discontinuity points of $G\left(\Lambda_{2} y\right)-G(y)+c$ are $\left\{\frac{a_{1}}{2}, a_{1}, 1-\frac{a_{1}}{2}\right\}$. Hence we have $a_{1}=1-\frac{a_{1}}{2}$ and $a_{1}=\frac{2}{3}$. Thus

$$
G(y)=c_{0} \cdot \mathbf{1}_{\left[0, \frac{2}{3}\right]}(y)+c_{1} \cdot \mathbf{1}_{\left[\frac{2}{3}, 1\right]}(y)
$$

and $F(y)$ has to be in the form of

$$
F(y)=\left(c_{1}-c_{0}\right) \cdot \mathbf{1}_{\left[\frac{1}{3}, \frac{2}{3}\right]}(y)+\left(c_{0}-c_{1}\right) \cdot \mathbf{1}_{\left[\frac{2}{3}, 1\right]}(y)+c,
$$

and $f(x)=b \cdot \mathbf{1}_{\left[-1,-\frac{1}{2}\right]}(x)-b \cdot \mathbf{1}_{\left[-\frac{1}{2}, \frac{1}{2}\right]}(x)+c$ with some constants $b, c$.

When $m=3$, by the similar argument as in the case $m=2$, we have $a_{1}=\frac{1}{3}$, $a_{2}=\frac{2}{3}$ and

$$
G(y)=c_{0} \cdot \mathbf{1}_{\left[0, \frac{1}{3}\right]}(y)+c_{1} \cdot \mathbf{1}_{\left[\frac{1}{3}, \frac{2}{3}\right]}(y)+c_{2} \cdot \mathbf{1}_{\left[\frac{2}{3}, 1\right]}(y) .
$$

Thus $G\left(\Lambda_{2} y\right)-G(y)$ has the form of

$$
\begin{aligned}
G\left(\Lambda_{2} y\right)-G(y)= & \left(c_{1}-c_{0}\right) \cdot \mathbf{1}_{\left[\frac{1}{6}, \frac{1}{3}\right]}(y)+\left(c_{2}-c_{1}\right) \cdot \mathbf{1}_{\left[\frac{1}{3}, \frac{2}{3}\right]}(y) \\
& +\left(c_{1}-c_{2}\right) \cdot \mathbf{1}_{\left[\frac{2}{3}, \frac{5}{6}\right]}(y)+\left(c_{0}-c_{2}\right) \cdot \mathbf{1}_{\left[\frac{5}{6}, 1\right]}(y) .
\end{aligned}
$$

Hence for $G\left(\Lambda_{2} y\right)-G(y)$ having 2-discontinuity points, we have $c_{2}-c_{1}=0$. It contradicts the assumption that $G(y)$ has 2-discontinuity points.

Remark 1. By exactly the same argument as in the proof of the case in Proposition 2, if $f(x)$ has only 1-discontinuity point, then it satisfies the Central Limit Theorem with respect to any Chebyshev polynomials of degree $l \geq 2$.

\section{REFERENCES}

1. R.L. Adler \& M.H. McAndrew: The entropy of Chebyshev polynomials. Trans. Amer. Math. Soc. 121 (1966), 236-241.

2. A. Boyarsky \& P. Góra: Laws of Chaos. Birkhäuser, 1997

3. G.H. Choe: Computational Ergodic Theory. Springer-Verlag, 2005.

4. W. Rudin: Real and Complex Analysis. McGraw-Hill, 1986.

5. P. Walters: An Introduction to Ergodic Theory. Springer-Verlag New York, 1982.

Department of Mathematics, Mokpo National University, Mokpo 534-729, Korea

Email address: yhahn@mokpo.ac.kr 